\title{
RETRACTED ARTICLE: Synthesis and characterization of Mn-doped CdS-diluted magnetic semiconductor nanoparticles
}

\author{
A. A. Gadalla ${ }^{1}$ A. N. Aboelkhir ${ }^{1}$ - M. G. Mahesha ${ }^{2} \cdot$ Ashok Rao $^{2}$
}

Received: 20 January 2020 / Accepted: 10 March 2020 / Published online: 29 March 2020

(c) Springer Science+Business Media, LLC, part of Springer Nature 2020

This article [1] has been retracted at the request of the authors. After publication, the authors realized that they accidentally submitted an early draft of their previously published paper [2]. As this article reports previously published results, this article is redundant. All authors agree to this Retraction. The online version of this article contains the full text of the retracted article as electronic supplementary material.

[1] Gadalla, A.A., Aboelkhir, A.N., Mahesha, M.G. et al. Synthesis and characterization of Mn-doped CdS-diluted magnetic semiconductor nanoparticles. J Mater Sci: Mater
Electron (2020). https://doi.org/10.1007/s10854-020-03240 $-\mathrm{X}$

[2] Gadalla, A.A., Almokhtar, M., Aboelkhir, A.N. Effect of Mn doping on structural, optical and magnetic properties of CdS diluted magnetic semiconductor nanoparticles. Chalcogenide Letters 14:4, 207-218 (2018)

Publisher's Note Springer Nature remains neutral with regard to jurisdictional claims in published maps and institutional affiliations.

Electronic supplementary material The online version of this article (https://doi.org/10.1007/s10854-020-03240-x) contains supplementary material, which is available to authorized users.

A. A. Gadalla

afaf.gadalla@science.au.edu.eg

$\triangle$ Ashok Rao

ashokanu_rao@rediffmail.com

1 Physics Department, Faculty of Science, Assiut University, Assiut, Egypt

2 Department of Physics, Manipal Institute of Technology, Manipal Academy of Higher Education, Manipal, Karnataka 576104, India 